NOTE

\title{
Radiochromic film dosimetry in water phantoms
}

\author{
Martin J Butson ${ }^{1,2,3}$, Tsang Cheung ${ }^{1}$ and Peter K N Yu${ }^{1}$ \\ ${ }^{1}$ City University of Hong Kong, Department of Physics and Materials Science, Kowloon Tong, \\ Hong Kong \\ 2 Illawarra Cancer Care Centre, Department of Medical Physics, Crown St, Wollongong, \\ NSW 2500, Australia \\ E-mail: mbutson@usa.net
}

Received 5 September 2000

\begin{abstract}
Radiochromic film is investigated for use in dosimetry in water phantoms as opposed to solid phantoms. Investigations are performed to measure the penetration rates of water into radiochromic film and to assess the effects on optical density that this penetration causes. The effects of film orientation during irradiation in water are also tested. Results show that only a small penetration rate is seen from water into the film which only affects the outer areas of the film, with penetration being less than $0.5 \mathrm{~mm}$ per hour. The optical density measurements of the film at $660 \mathrm{~nm}$ remain unchanged in the unaffected regions of the radiochromic film. Minimal effects are seen due to beam orientation in a water phantom as opposed to solid water phantoms in which an overestimation in dose is normally seen for parallel irradiation. Radiochromic film seems to be an adequate detector for dosimetry in a water phantom where high spatial resolution is needed and angle of beam incidence at the point of interest is important.
\end{abstract}

\section{Introduction}

Film dosimetry with radiochromic film (Gafchromic MD-55-2) has become an attractive alternative to dosimetry with radiographic film due to properties such as energy dependence, automatic development and physical toughness. It is also a useful alternative to thermoluminescent dosimeters (TLDs) due to its spatial resolution and ease of readout. Radiochromic film lends itself to dosimetry in volumetric regions due to its flexible nature and high spatial resolution. That is, it can be curled around an area of interest for dose assessment; this can be done around a solid phantom or in a water phantom. Work with Gafchromic film in solid phantom materials has been reported previously (Klassen et al 1997, Butson et al 1998, Meigooni et al 1996), but quantitative analysis of the effects of water are not quoted in the literature. Dosimetry in this manner raises two issues: (a) the effects of water during exposure for film dosimetry (neither TLDs nor radiographic film are suitable for water dosimetry without

\footnotetext{
${ }^{3}$ Address for correspondence: Department of Medical Physics, PO Box 1798, Wollongong 2500 NSW, Australia.
} 
some sort of protective coating being placed over them) and (b) the response of the film due to orientation with respect to the beam direction in water as opposed to solid phantoms. Both of these effects are examined in this paper for MD-55-2 radiochromic film.

\section{Materials and methods}

The film used was Gafchromic MD-55-2, lot number H1146MD55. Following the AAPM TG-55 recommendations (Niroomand-Rad et al 1998), appropriate precautions in handling, calibrating and scanning the radiochromic film were taken. The film results were analysed using a double-exposure technique (Meigooni et al 1996). This is performed by giving each film an initial dose of $5 \mathrm{~Gy}$ to ascertain if any corrections are needed due to non-uniformity in dose response. A variation in initial optical density of the film of $9 \%$ was recorded for the films used in the experiment. This represents the non-uniformity of response for the unirradiated film. The film was analysed with an in-house built densitometer which has a $660 \mathrm{~nm}, 1000 \mathrm{mcd}$ GaAlAs LED light source for film analysis. Negligible polarization effects were observed using this densitometer. The film was left for a period of $24 \mathrm{~h}$ after exposure prior to optical density measurements to reduce the effects of post-irradiation coloration (Muench et al 1991, Reinstein et al 1997). Gafchromic film should be handled using either soft gloves or tweezers to avoid finger prints and other contaminants which affect readout. By attaching a paper or plastic tab onto the side of the film with adhesive tape it can also be easily handled without touching the film. Gafchromic film is prone to scratching which can also affect the optical density readout, and care should be taken not to slide the film on surfaces with any force.

Tests were performed to evaluate the dosimetric qualities of Gafchromic film in water phantoms. The film was cut with the use of scissors into $3 \mathrm{~cm} \times 3 \mathrm{~cm}$ squares and placed in water to assess the penetration rate into the film and any effects on dosimetric properties of the film after this penetration. Films were left for up to 2 days in a temperature controlled water bath with measurements made of optical density of the film and amount of visible water penetration into the side of the film at regular intervals. The water baths were maintained at temperatures ranging from $15^{\circ} \mathrm{C}$ to $30^{\circ} \mathrm{C}$, which was considered a normal range of working temperatures. The films were also exposed to $\mathrm{x}$-rays after soaking for various lengths of time and measurements were made of optical density to assess any differences in film response.

Measurements were also made to assess the dosimetric sensitivity of radiochromic film as a function of radiation beam incidence in water. The film was placed at $D_{\max }$ and at $10 \mathrm{~cm}$ depth and irradiated with $6 \mathrm{MV}$ x-rays with the film orientated at various angles from perpendicular to parallel to the beam direction. This was performed in both an RMI solid water phantom and a water phantom. The film used in the water phantom had not received any prior soaking in water. Depth dose curves were also measured for a $6 \mathrm{MV}$ x-ray, $10 \mathrm{~cm} \times 10 \mathrm{~cm}$ field size at $100 \mathrm{~cm}$ SSD in both perpendicular and parallel configurations in water.

\section{Results and discussion}

Figure 1 shows the average visible penetration rate of water into the edges of the Gafchromic film as a function of time. Water penetration into the edge of the film produced an opaque, whitish colour and the values quoted are the visible point at which the film seemed to retain its original colour and transparency. The visible change seen in opacity for the water-penetrated film remains for an extended period of time. As the water evaporates from the film edges the whitish region turns a darker blue colour than the original blue colour of the film. This was noticed approximately 7-10 days after the film was permanently removed from the water 


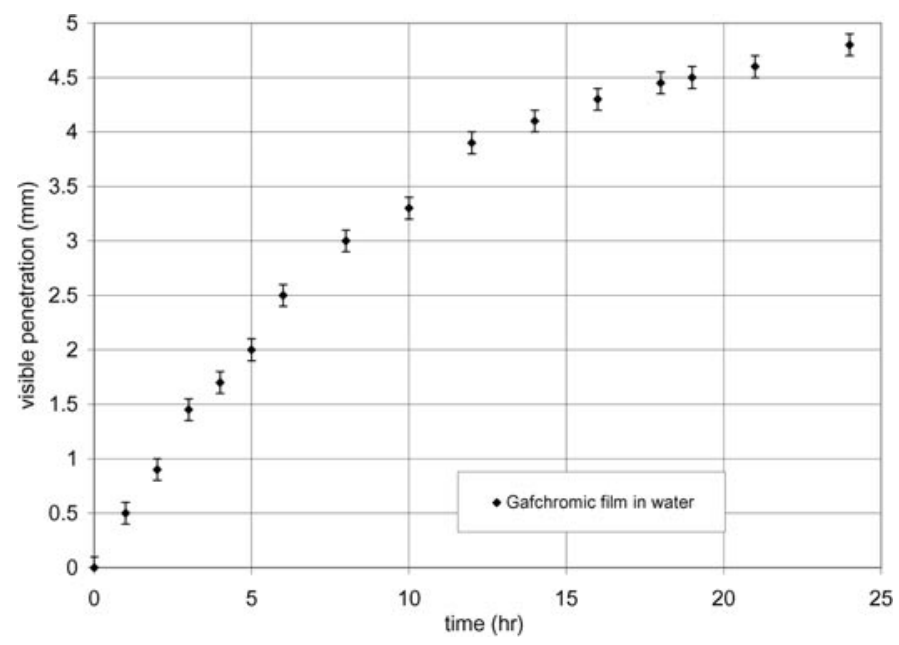

Figure 1. Penetration of water into the edges of Gafchromic film with time.

Table 1. Measured optical density values for radiochromic film after water exposure.

\begin{tabular}{cll}
\hline & \multicolumn{2}{c}{ Optical density } \\
\cline { 2 - 3 } Time (h) & at 0 Gy & at 10 Gy \\
\hline 0 & 0.152 & 0.721 \\
1 & 0.156 & 0.734 \\
2 & 0.149 & 0.710 \\
5 & 0.154 & 0.729 \\
10 & 0.152 & 0.727 \\
16 & 0.149 & 0.720 \\
24 & 0.153 & 0.730 \\
\hline
\end{tabular}

bath. No obvious variations in the penetration rate were seen within the temperature range of $15^{\circ} \mathrm{C}$ up to $30^{\circ} \mathrm{C}$. The errors quoted are one standard deviation of the mean value for five film pieces and with measurement taken on all four sides of the square film piece. The water seems to penetrate at a slightly decreasing rate with time. During this period the optical density of the film was measured at regular intervals. This was performed by taking the film out of the water and towel drying it before the reading. The optical density at the centre of the film remained unchanged within experimental errors. A slight change $(3 \%-5 \%)$ was measured up to approximately $2 \mathrm{~mm}$ inside the visible edge of the water penetration mark but no measurable change was found beyond this point. This change is probably due to the fact that the visible change seen by eye is not as accurate as that measured with a densitometer. These results show that radiochromic film is not significantly affected by placement in water during experimental exposure. Normally an experimental procedure for dosimetry with radiochromic film in radiotherapy should take at most $1 \mathrm{~h}$. This time would include set up and irradiation. Thus only $0.5 \mathrm{~mm}$ of the edge of the radiochromic film would be damaged by water during the process and no significant effects on dosimetry are expected. Thus MD-55-2 can be used in a water phantom for dose assessment.

Table 1 shows optical density versus time submerged in water prior to irradiation readings for unirradiated and irradiated Gafchromic film. These values are the OD levels measured at the centre of each piece of film. No systematic changes in optical density were seen with respect 


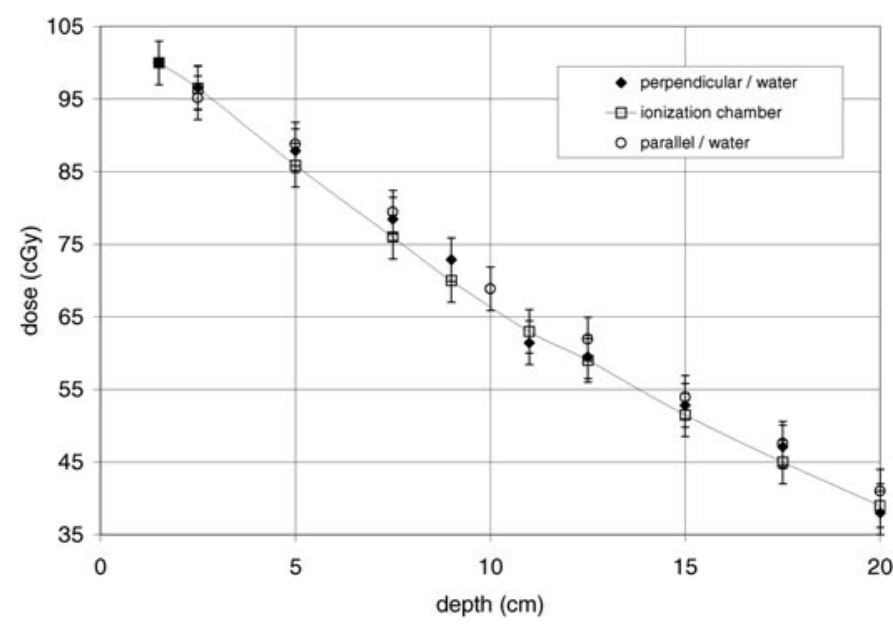

Figure 2. Percentage depth dose curve for Gafchromic film in a water phantom for parallel and perpendicular exposure in combination with ionization chamber results.

Table 2. Response of Gafchromic film at various angles of incidence in water and solid water.

\begin{tabular}{llllll}
\hline \multirow{2}{*}{$\begin{array}{l}\text { Angle of incidence } \\
\text { deg) }\end{array}$} & \multicolumn{2}{c}{ Water } & & \multicolumn{2}{c}{ Solid water } \\
\cline { 2 - 3 } \cline { 5 - 6 } & $D_{\max }$ & $10 \mathrm{~cm}$ & & $D_{\max }$ & $10 \mathrm{~cm}$ \\
\hline 90 & 1.000 & 1.000 & & 1.000 & 1.000 \\
45 & 1.023 & 0.974 & & 1.011 & 1.032 \\
30 & 1.014 & 0.993 & & 1.018 & 1.005 \\
15 & 0.982 & 1.014 & & 0.992 & 0.983 \\
3 & 0.990 & 0.991 & & 0.981 & 1.021 \\
0 & 0.985 & 0.991 & & 0.977 & 1.052 \\
\hline
\end{tabular}

to time when the film was placed in the water bath. Table 2 shows the normalized results for irradiations performed with various orientation angles of the film, from perpendicular $\left(90^{\circ}\right)$ to parallel $\left(0^{\circ}\right)$ with respect to the beam central axis. Results are normalized to 1 for perpendicular exposure in each case. There is a minimal sensitivity effect for Gafchromic film due to perpendicular or parallel irradiation in water phantoms. A slightly larger effect is seen in solid water with parallel exposure but this is minimized with a $3^{\circ}$ rotation. The over response of the film for parallel exposure in solid water is assumed to be due mainly to the presence of air gaps which are created when using thin slices of film for dosimetry, as with radiographic film (Suchowerska et al 1999). These results highlight that water would be the optimal medium for dosimetry, to remove any spurious results caused by imperfect film-phantom contact during parallel film exposure which could occur with the use of solid phantom materials.

Figure 2 shows depth dose curves for a $10 \mathrm{~cm} \times 10 \mathrm{~cm}$ field size as measured by Gafchromic film in a water phantom for parallel and perpendicular irradiations. Results are the average of five film experiments with errors being one standard deviation from the mean. Also shown are results for ionization chamber measurements in the same beam configuration. Results for Gafchromic film are corrected using the double-exposure technique. No corrections for sensitivity changes at depth are made to these curves. The Gafchromic film adequately matches measurement of dose at depth with the ionization chamber in both configurations. These results show that the orientation of the film or the effects of water on the film do not significantly 
affect dosimetry. With this in mind, more complicated dosimetry over a curved area in a water phantom could be performed without the danger of deviation in results caused by water damage or film orientation.

\section{Conclusion}

Gafchromic MD-55-2 film provides an adequate dosimetry tool for measurement of dose in a water or solid water phantom. The effects of water on the film are minimal. Results at the edge of the film should be disregarded where visible penetration has occurred but other areas within the film are not affected when the film is placed in a water phantom for dosimetry purposes. The film also has minimal variations in sensitivity with orientation or depth in a water phantom.

\section{Acknowledgments}

This research was performed with the financial assistance from grants 7100066 and 9040545 from the City University of Hong Kong.

\section{References}

Butson M, Yu P K N and Metcalfe P 1998 Measurement of off axis and peripheral skin dose using radiochromic film Phys. Med. Biol. 43 2647-50

Klassen N, Zwan L and Cygler J 1997 GafChromic MD-55: investigated as a precision dosimeter Med. Phys. 24 1924-34

Meigooni A S, Sanders M, Ibbott G and Szeglin 1996 Dosimetric characteristics of improved radiochromic film Med. Phys. 23 1883-8

Muench P J, Meigooni A S, Nath R and McLaughlin W L 1991 Photon energy dependence of the sensitivity of radiochromic film and comparison with silver halide film and LiF TLDs used for brachytherapy dosimetry Med. Phys. 18 769-75

Niroomand-Rad A et al 1998 Radiochromic film dosimetry: Recommendation of AAPM radiation therapy task group 55 Med. Phys. 25 2093-115

Reinstein L, Gluckman G, Pai S and Meek A 1997 Post irradiation dependence of radiochromic film optical density on temperature and time Med. Phys. 24 1005-9

Suchowerska N, Hoban P, Davison A and Metcalfe P 1999 Perturbation of radiotherapy beams by radiographic film: measurements and Monte Carlo simulations Phys. Med. Biol. 44 1755-65 